\title{
Giant saphenous vein graft aneurysm compressing the lingular bronchus
}

\author{
Peter Chiu, MD, Itai Palmon, and Michael P. Fischbein, MD, PhD, Stanford, Calif
}

\footnotetext{
From the Department of Cardiothoracic Surgery, Stanford University, School of Medicine, Stanford, Calif. Support by a KL2 Mentored Career Development Award of the Stanford Clinical and Translational Science Award to Spectrum (NIH KL2 TR 001083).

Disclosures: M.F. has received speaking honoraria from St Jude and Edwards Lifesciences and receives funding from the National Institutes of Health (NIH R01AR066629-01A1). All other authors have nothing to disclose with regard to commercial support.

Received for publication July 11, 2016; revisions received Aug 26, 2016; accepted for publication Aug 30, 2016; available ahead of print Oct 19, 2016.

Address for reprints: Michael P. Fischbein, MD, PhD, 300 Pasteur Dr, Falk CVRB ULN MC5407, Stanford, CA 94305 (E-mail: mfischbe@stanford.edu).

J Thorac Cardiovasc Surg 2017;153:e1-3

$0022-5223 / \$ 36.00$

Copyright (C) 2016 by The American Association for Thoracic Surgery

http://dx.doi.org/10.1016/j.jtcvs.2016.08.062
}

Saphenous vein graft (SVG) aneurysm is a rare complication of coronary artery bypass grafting, occurring in approximately $0.07 \%$ of grafts in a single-center retrospective study. ${ }^{1}$ Operative intervention for SVG aneurysm has been associated with perioperative mortality as great as $13.9 \%$, although mortality may be higher among conservatively treated patients. ${ }^{2}$ We present the case of a patient with a giant SVG aneurysm that caused compression of the lingular bronchus and was repaired through a left thoracotomy.

\section{CLINICAL SUMMARY}

A 47-year-old gentleman had a non-ST elevation myocardial infarction and subsequently underwent 4vessel coronary artery bypass grafting in 1992. In 2005, the patient began to have angina; cardiac catheterization demonstrated a focal high-grade lesion in the proximal portion of the SVG to the obtuse marginal and ectasia distally, and a coronary stent was placed. His symptoms improved, but in 2011 he was seen with dyspnea on exertion and positional shortness of breath. Repeated cardiac catheterization demonstrated an SVG aneurysm distal to the stent; the aneurysm measured $49 \times 52 \mathrm{~mm}$ on computed tomographic (CT) angiography. Because of his primarily respiratory symptoms, the patient was seen by a pulmonologist. Pulmonary function tests suggested a bronchodilator-responsive obstructive ventilatory defect, and therapy was initiated with fluticasone and salmeterol. Follow-up CT revealed an interval increase in size to $79 \times 71 \mathrm{~mm}$, with additional extrinsic compression of the lingular bronchus (Figure $1, A$ and $B$ ). The SVG aneurysm was visible by transthoracic echocardiography (Figure 1, C). Stress echocardiography

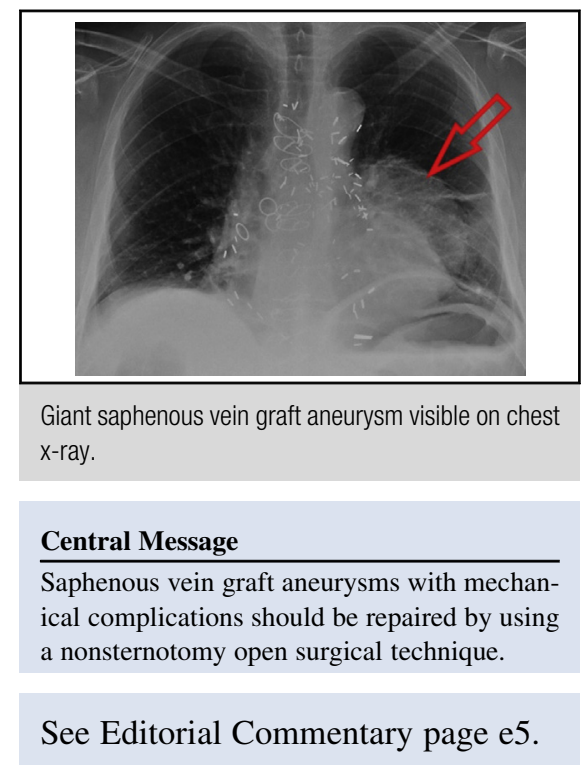

demonstrated an ejection fraction of $36 \%$ and an inducible wall motion abnormality of the anterior wall. Repeated coronary angiography, however, demonstrated a patent left internal thoracic artery graft to the left anterior descending artery and no suitable targets for revascularization. The decision was made to manage his ischemic cardiomyopathy medically and operate on the giant aneurysm. Preoperative chest radiography demonstrated a large opacity along the left heart border (Figure 2, $A$ and $B$ ).

The patient was brought to the operating room, and an epidural catheter was placed. General anesthesia was induced, and a dual-lumen endotracheal tube was placed. A left anterolateral thoracotomy was performed through the fifth intercostal space. Extensive adhesions were encountered within the pericardium, and neither proximal nor distal control was obtainable; moreover, external ligation was not possible. After systemic heparinization, the aneurysm was entered and found to be full of laminated, organized clot. The inflow to the aneurysm was occluded with an $8 \mathrm{~F}$ balloon occlusion catheter (Figure 2, C). Bovine pericardial patches were fashioned to cover both the proximal and distal limbs of the SVG (Figure 2, D). The thoracotomy was closed in the usual fashion, and the patient was extubated in the operating room. 

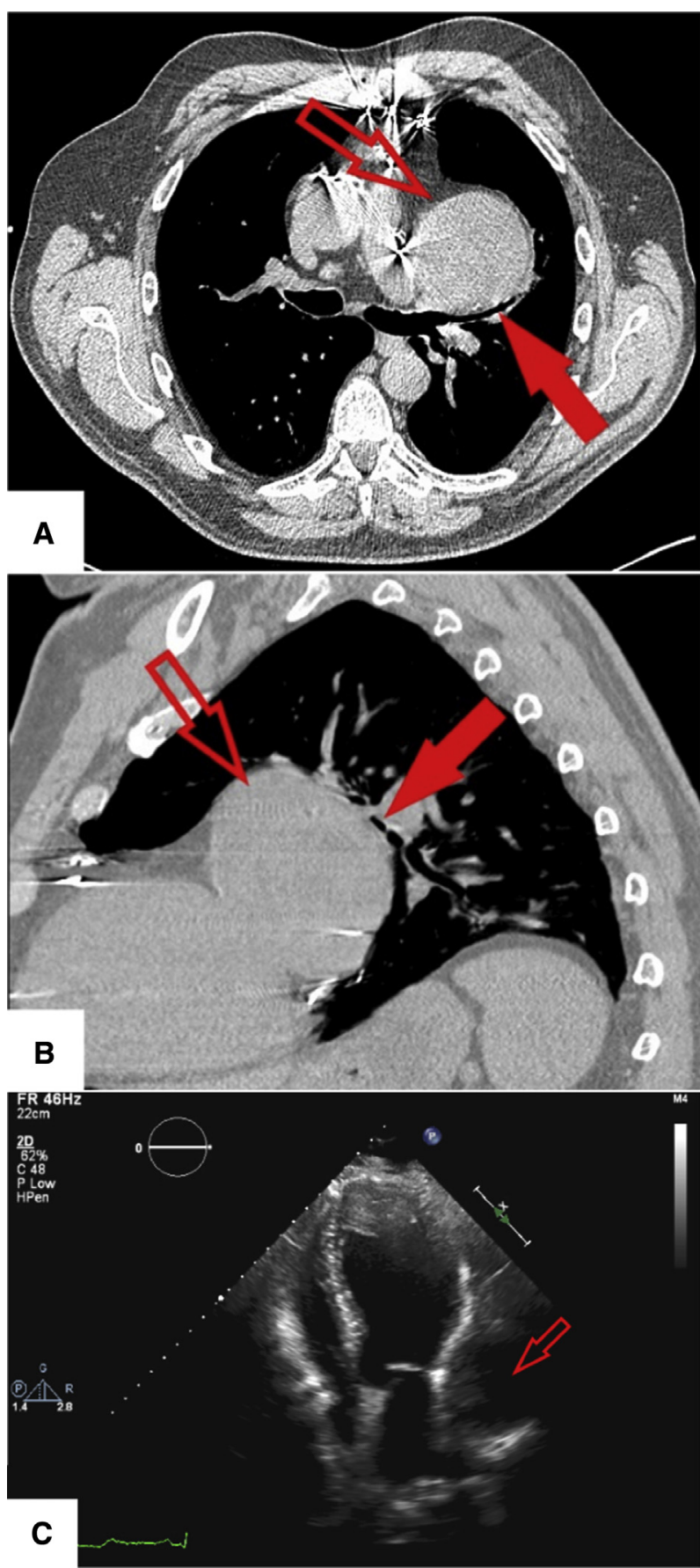

FIGURE 1. A, Noncontrast axial computed tomography of $79 \times 71-\mathrm{mm}$ saphenous vein graft aneurysm compressing the lingular bronchus. B, Noncontrast sagittal computed tomographic reconstruction of saphenous vein graft aneurysm. C, Apical 4-chamber echocardiographic view demonstrating the presence of a saphenous vein graft aneurysm along the atrioventricular groove. Hollow red arrows mark the saphenous vein graft aneurysm; solid red arrows indicate compression of the lingular bronchus.
The patient was transferred to the step-down unit on postoperative day 1 and discharged home on postoperative day 7. Follow-up CT angiography demonstrated flow into the distal portion of the SVG through collaterals; the proximal limb of the SVG was thrombosed. The patient's ischemic cardiomyopathy has been medically optimized. He was last seen in the cardiology clinic at 29 months postoperatively, at which time he was doing well and had improved respiratory symptoms.

\section{DISCUSSION}

SVG aneurysms are rare but are being reported with greater frequency. ${ }^{3}$ Whether this is due to alterations in technique, longer follow-up of patients undergoing coronary artery bypass grafting, or improved access to and increased use of advanced imaging is unknown. Whereas true aneurysms have been attributed to degenerative processes, pseudoaneurysms - representing as many as $30 \%$ of aneurysmal lesions-are more commonly attributed to technical issues. ${ }^{2,3}$ Regardless of the etiology, an aging population of patients with aortocoronary SVGs necessitates greater awareness of this process.

Open surgical repair has been the strategy most commonly reported, but endovascular methods have emerged as a viable alternative. ${ }^{2}$ In patients with mechanical complications, an open approach is advisable. Thoracotomy may be preferable to resternotomy because of the potential for either disrupting a patent internal thoracic artery graft or requiring extensive mobilization to reach the lesion. As such, advanced axial imaging with CT to evaluate the size and location is critical for planning the optimal approach. In patients who require distal revascularization, aneurysmectomy along with a Dacron polyester or saphenous vein interposition graft may be used. Alternatively, aortocoronary bypass from either the ascending or descending aorta may also be considered depending on the operative exposure.

\section{References}

1. Dieter RS, Patel AK, Yandow D, Pacanowski JP Jr, Bhattacharya A, Gimelli G, et al. Conservative vs. invasive treatment of aortocoronary saphenous vein graft aneurysms: treatment algorithm based upon a large series. Cardiovasc Surg. 2003;11:507-13.

2. Ramirez FD, Hibbert B, Simard T, Pourdjabbar A, Wilson KR, Hibbert R, et al. Natural history and management of aortocoronary saphenous vein graft aneurysms: a systematic review of published cases. Circulation. 2012;126: 2248-56.

3. Memon AQ, Huang RI, Marcus F, Xavier L, Alpert J. Saphenous vein graft aneurysm: case report and review. Cardiol Rev. 2003;11:26-34. 


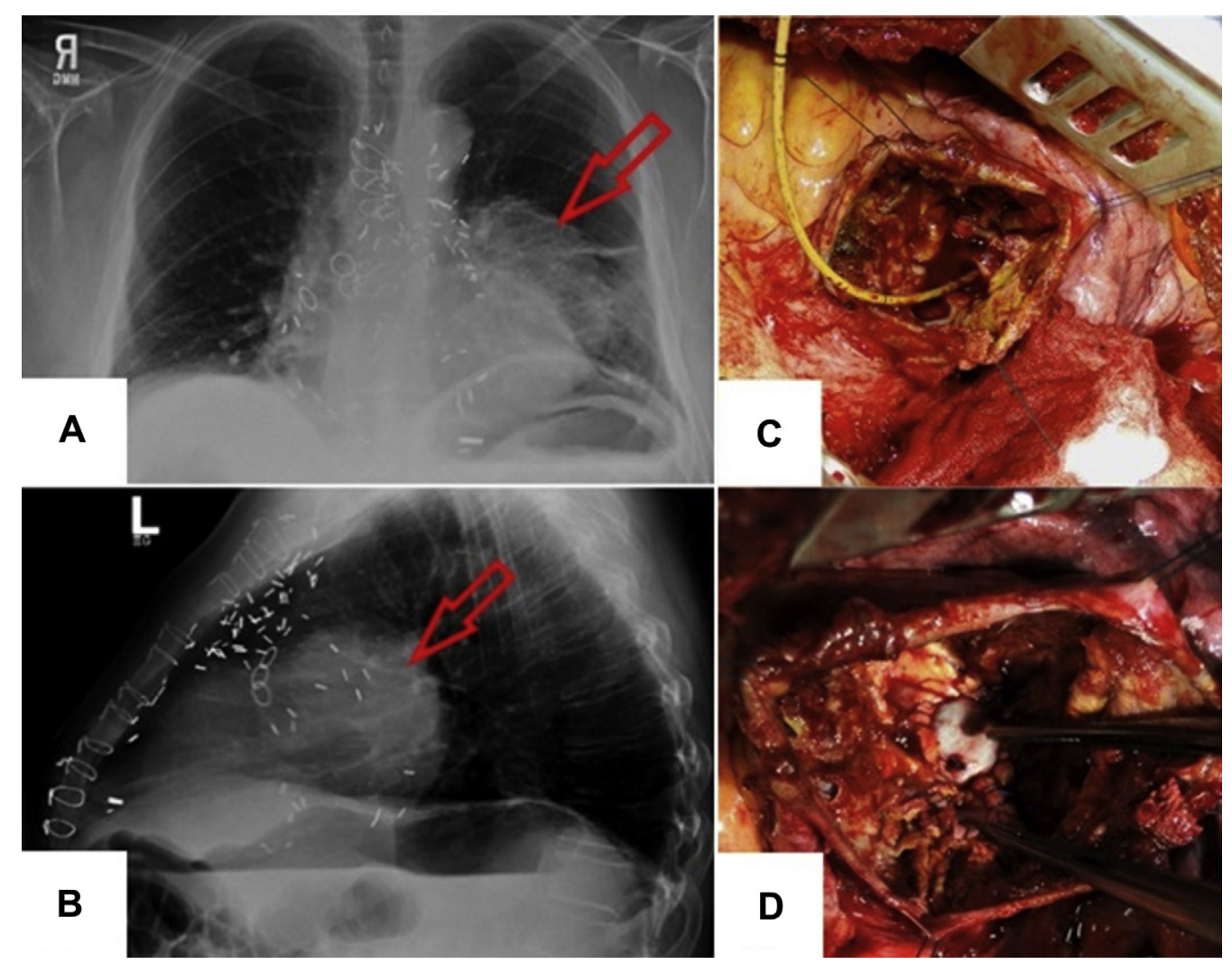

FIGURE 2. Two-view chest radiography demonstrates a large, well-circumscribed mass along the left heart border in the posteroanterior view (A) and the lateral view (B). Hollow red arrows mark the saphenous vein graft aneurysm. Intraoperative photographs of saphenous vein graft aneurysm repair show exposure of the aneurysm's inflow with balloon occlusion catheter in situ (C) and the proximal patch occlusion of the saphenous vein graft (D). 